\title{
A New Proof to the Necessity of a Second Moment Stability Condition of Discrete-Time Markov Jump Linear Systems with Real States
}

\author{
Qiang Ling ${ }^{1}$ and Haojiang Deng ${ }^{2}$ \\ ${ }^{1}$ Department of Automation, University of Science and Technology of China, Anhui, Hefei 230027, China \\ ${ }^{2}$ National Network New Media Engineering Research Center, Institute of Acoustics, \\ Chinese Academy of Science, Beijing 100190, China
}

Correspondence should be addressed to Qiang Ling, qling@ustc.edu.cn

Received 12 February 2012; Accepted 28 March 2012

Academic Editor: Baocang Ding

Copyright (C) 2012 Q. Ling and H. Deng. This is an open access article distributed under the Creative Commons Attribution License, which permits unrestricted use, distribution, and reproduction in any medium, provided the original work is properly cited.

This paper studies the second moment stability of a discrete-time jump linear system with real states and the system matrix switching in a Markovian fashion. A sufficient stability condition was proposed by Fang and Loparo (2002), which only needs to check the eigenvalues of a deterministic matrix and is much more computationally efficient than other equivalent conditions. The proof to the necessity of that condition, however, is a challenging problem. In the paper by Costa and Fragoso (2004), a proof was given by extending the state domain to the complex space. This paper proposes an alternative necessity proof, which does not need to extend the state domain. The proof in this paper demonstrates well the essential properties of the Markov jump systems and achieves the desired result in the real state space.

\section{Introduction}

\subsection{Background of the Discrete-Time Markov Jump Linear Systems}

This paper studies the stability condition of discrete-time jump linear systems in the real state domain. In a jump linear system, the system parameters are subject to abrupt jumps. We are concerned with the stability condition when these jumps are governed by a finite Markov chain. A general model is shown as follows:

$$
\begin{aligned}
& x[k+1]=A[q[k]] x[k], \\
& x[0]=x_{0}, \quad q[k]=q_{0},
\end{aligned}
$$


where $x[k] \in R^{n}$ is the state and $\{q[k]\}$ is a discrete-time Markov chain with a finite state space $\left\{q_{1}, q_{2}, \ldots, q_{N}\right\}$ and a transition matrix $Q=\left(q_{i j}\right)_{N \times N^{\prime}}$, where $q_{i j}=P\left(q[k+1]=q_{j} \mid q[k]=\right.$ $\left.q_{i}\right) . x_{0} \in R^{n}$ is the initial state. $q_{0}$ is the initial Markov state, whose distribution is denoted as $p=\left[\begin{array}{llll}p_{1} & p_{2} & \cdots & p_{N}\end{array}\right]$ with $p_{i}=P\left(q_{0}=q_{i}\right) .\{q[k]\}$ is assumed to be a time-homogeneous aperiodic Markov chain. When $q[k]=q_{i}, A[q[k]]=A_{i}(i=1, \ldots, N)$, that is, $A[q[k]]$ switches among $\left\{A_{i}\right\}_{i=1}^{N}$. A compound matrix is constructed from $A_{i}$ as

$$
A_{[2]}=\left(Q^{T} \otimes I_{n^{2}}\right) \operatorname{diag}\left(A_{i} \otimes A_{i}\right)_{i=1}^{N},
$$

where $I_{n^{2}}$ denotes an identity matrix with the order of $n^{2}$ and $\otimes$ denotes the Kronecker product [1]. A brief introduction on the Kronecker product will be given in Section 2.1.

For the jump linear system in (1.1), the first question to be asked is "is the system stable?" There has been plenty of work on this topic, especially in 90s, [2-6]. Recently this topic has caught academic interest again because of the emergence of networked control systems [7]. Networked control systems often suffer from the network delay and dropouts, which may be modelled as Markov chains, so that networked control systems can be classified into discrete-time jump linear systems [8-11]. Therefore, the stability of the networked control systems can be determined through studying the stability of the corresponding jump linear systems. Before proceeding further, we review the related work.

\subsection{Related Work}

At the beginning, the definitions of stability of jump linear systems are considered. In [6], three types of second moment stability are defined.

Definition 1.1. For the jump linear system in (1.1), the equilibrium point 0 is

(1) stochastically stable, if, for every initial condition $\left(x[0]=x_{0}, q[0]=q_{0}\right)$,

$$
\mathrm{E}\left[\sum_{k=0}^{\infty}\|x[k]\|^{2} \mid x_{0}, q_{0}\right]<\infty,
$$

where $\|\cdot\|$ denotes the 2-norm of a vector;

(2) mean square stable (MSS), if, for every initial condition $\left(x_{0}, q_{0}\right)$,

$$
\lim _{k \rightarrow \infty} \mathbf{E}\left[\|x[k]\|^{2} \mid x_{0}, q_{0}\right]=0
$$

(3) exponentially mean square stable, if, for every initial condition $\left(x_{0}, q_{0}\right)$, there exist constants $0<\alpha<1$ and $\beta>0$ such that for all $k \geq 0$,

$$
\mathrm{E}\left[\|x[k]\|^{2} \mid x_{0}, q_{0}\right]<\beta \alpha^{k}\left\|x_{0}\right\|^{2},
$$

where $\alpha$ and $\beta$ are independent of $x_{0}$ and $q_{0}$. 
In [6], the above 3 types of stabilities are proven to be equivalent. So we can study mean square stability without loss of generality. In [6], a necessary and sufficient stability condition is proposed.

Theorem 1.2 (see [6]). The jump linear system in (1.1) is mean square stable, if and only if, for any given set of positive definite matrices $\left\{W_{i}: i=1, \ldots, N\right\}$, the following coupled matrix equations have unique positive definite solutions $\left\{M_{i}: i=1, \ldots, N\right\}$ :

$$
\sum_{j=1}^{N} q_{i j} A_{i}^{T} M_{j} A_{i}-M_{i}=-W_{i}
$$

Although the above condition is necessary and sufficient, it is difficult to verify because it claims validity for any group of positive definite matrices $\left\{W_{i}: i=1, \ldots, N\right\}$. A more computationally efficient testing criterion was, therefore, pursued [3, 4, 12-15]. Theorem 1.3 gives a sufficient mean square stability condition.

Theorem 1.3 (see [4, 12]). The jump linear system in (1.1) is mean square stable, if all eigenvalues of the compound matrix $A_{[2]}$ in (1.2) lie within the unit circle.

Remark 1.4. By Theorem 1.3, the mean square stability of a jump linear system can be reduced to the stability of a deterministic system in the form $y_{k+1}=A_{[2]} y_{k}$ [13]. Thus the complexity of the stability problem is greatly reduced. Theorem 1.3 only provides a sufficient condition for stability. The condition was conjectured to be necessary as well $[2,15]$. In the following, we briefly review the research results related to Theorem 1.3.

In [14], Theorem 1.3 was proven to be necessary and sufficient for a scalar case, that is, $A_{i}(i=1, \ldots, N)$ are scalar. In [15], the necessity of Theorem 1.3 was proven for a special case with $N=2$ and $n=2$. In [4,12], Theorem 1.3 was asserted to be necessary and sufficient for more general jump linear systems. Specifically, Bhaurucha [12] considered a random sampling system with the sampling intervals governed by a Markov chain while Mariton [4] studied a continuous-time jump linear system. Although their sufficiency proof is convincing, their necessity proof is incomplete.

The work in [3] may shed light on the proof of the necessity of Theorem 1.3. In [3], a jump linear system model being a little different from (1.1) is considered. The difference lies in

(i) $x[k] \in C^{n}$, where $C$ stands for the set of complex numbers,

(ii) $x_{0} \in S_{c}$, where $S_{c}$ is the set of complex vectors with finite second-order moments in the complex state space.

The mean square stability in [3] is defined as

$$
\operatorname{Lim}_{k \rightarrow \infty} \mathrm{E}\left[x[k] x^{*}[k] \mid x_{0}, q_{0}\right]=0, \quad \forall x_{0} \in S_{c}, \forall q_{0},
$$




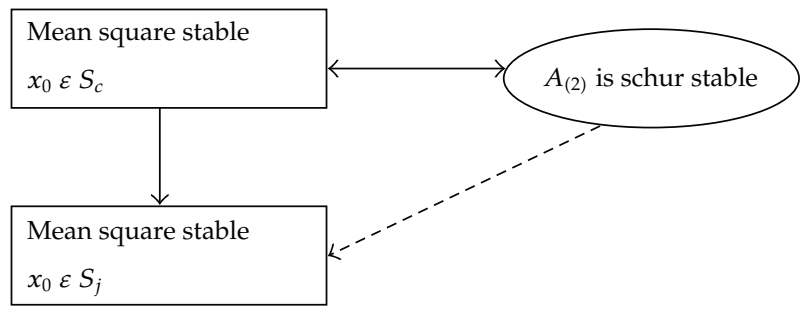

Figure 1: Relationship between different senses of mean square stability.

where ${ }^{*}$ stands for the conjugate transpose. Corresponding to the definition in (1.7), the mean square stability in (1.4) can be eewritten into (because $x[k] \in R^{n}$ in (1.4), there is no difference between $x^{T}[k]$ and $\left.x^{*}[k]\right)$,

$$
\operatorname{Lim}_{k \rightarrow \infty} \mathrm{E}\left[x[k] x^{*}[k] \mid x_{0}, q_{0}\right]=0, \quad \forall x_{0} \in S_{j}, \forall q_{0}
$$

where $S_{j}$ is the set of all vectors in $R^{n}$. For any vector $x \in R^{n}$, we can treat it as a random vector with a single element in $R^{n}$, and also a random vector in $C^{n}$. Of course, such random vectors have finite second-order moments. Therefore, we know

$$
S_{j} \subset S_{c}, \quad S_{j} \cap S_{c} \neq S_{c} .
$$

It can be seen that the mean square stability in (1.7) requires stronger condition $\left(x_{0} \in S_{c}\right)$ than the one in $(1.8)\left(x_{0} \in S_{j}\right)$. When $A_{i}(i=1, \ldots, N)$ are real matrices, a necessary and sufficient stability condition was given in the complex state domain.

Theorem 1.5 (see [3]). The jump linear system in (1.1) (with complex states) is mean square stable in the sense of (1.7) if and only if $A_{[2]}$ is Schur stable.

Due to the relationship of $S_{j} \subset S_{c}$ and Theorem 1.5, we can establish the relationship diagram in Figure 1. As it shows, the Schur stability of $A_{[2]}$ is a sufficient condition for mean square stability with $x_{0} \in S_{j}$ at the first look.

We are still wondering "whether the condition in Theorem 1.3 is necessary too?" the answer is definitely "yes." That necessity was conjectured in [2]. A proof to the necessity of that condition was first given in [16], which extends the state domain to the complex space and establishes the desired necessity in the stability sense of (1.7). As mentioned before, our concerned stability (in the sense of (1.8)) is weaker than that in (1.7). This paper proves that the weaker condition in (1.8) still yields the schur stability of $A_{[2]}$, that is, the necessity of theorem 1.3 is confirmed. This paper confines the state to the real space domain and makes the best use of the essential properties of the markov jump linear systems to reach the desired necessity goal. In Section 2, a necessary and sufficient version of Theorem 1.3 is stated and its necessity is strictly proven. In Section 3, final remarks are placed. 


\section{A Necessary and Sufficient Condition for Mean Square Stability}

This section will give a necessary and sufficient version of Theorem 1.3. Throughout this section, we will define mean square stability in the sense of $(1.4)\left(x_{0} \in S_{j}\right)$. At the beginning, we will give a brief introduction to the Kronecker product and list some of its properties. After then, the main result, a necessary and sufficient condition for the mean square stability, is presented in Theorem 2.1 and its necessity is proven by direct matrix computations.

\subsection{Mathematical Preliminaries}

Some of the technical proofs in this paper make use of the Kronecker product, $\otimes$ [1]. The Kronecker product of two matrices $A=\left(a_{i j}\right)_{M \times N}, B=\left(b_{p q}\right)_{P \times Q}$ is defined as

$$
A \otimes B=\left[\begin{array}{cccc}
a_{11} B & a_{12} B & \cdots & a_{1 N} B \\
a_{21} B & a_{22} B & \cdots & a_{2 N} B \\
\vdots & \vdots & \ddots & \vdots \\
a_{M 1} B & a_{M 2} B & \cdots & a_{M N} B
\end{array}\right]_{M P \times N Q}
$$

For simplicity, $A \otimes A$ is denoted as $A^{[2]}$ and $A \otimes A^{[n]}$ is denoted as $A^{[n+1]}(n \geq 2)$.

For two vectors $x$ and $y, x \otimes y$ simply rearranges the columns of $x y^{T}$ into a vector. So for two stochastic processes $\{x[n]\}$ and $\{y[n]\}, \lim _{n \rightarrow \infty} \mathbf{E}[x[n] \otimes y[n]]=0$ if and only if $\lim _{n \rightarrow \infty} \mathbf{E}\left[x[n] y^{T}[n]\right]=0$. Furthermore, if $\lim _{n \rightarrow \infty} \mathbf{E}\left[x^{[2]}[n]\right]=0$ and $\lim _{n \rightarrow \infty} \mathbf{E}\left[y^{[2]}[n]\right]=0$, then

$$
\lim _{n \rightarrow \infty} \mathbf{E}[x[n] \otimes y[n]]=0 .
$$

The following property of the Kronecker product will be frequently used in the technical proofs

$$
\left(A_{1} A_{2} \cdots A_{n}\right) \otimes\left(B_{1} B_{2} \cdots B_{n}\right)=\left(A_{1} \otimes B_{1}\right)\left(A_{2} \otimes B_{2}\right) \cdots\left(A_{n} \otimes B_{n}\right),
$$

where $A_{i}, B_{i}(i=1,2, \ldots, n)$ are all matrices with appropriate dimensions.

Our computations need two linear operators, vec and devec. The vec operator transforms a matrix $A=\left(a_{i j}\right)_{M \times N}$ into a vector as

$$
\operatorname{vec}(A)=\left[a_{11} \cdots a_{M 1} a_{12} \cdots a_{M 2} \cdots a_{1 N} \cdots a_{M N}\right]^{T} .
$$

The devec operator inverts the vec operator for a square matrix, that is,

$$
\operatorname{devec}(\operatorname{vec}(A))=A,
$$

where $A$ is a square matrix. 


\subsection{Main Results}

Theorem 2.1. The jump linear system in (1.1) is mean square stable if and only if $A_{[2]}$ is Schur stable, that is, all eigenvalues of $A_{[2]}$ lie within the unit circle.

There are already some complete proofs for sufficiency of Theorem 2.1, [3, 12, 13]. So we will focus on the necessity proof. Throughout this section, the following notational conventions will be followed.

The initial condition of the jump linear system in (1.1) is denoted as $x[0]=x_{0}, q[0]=q_{0}$ and the distribution of $q_{0}$ is denoted as $p=\left[\begin{array}{llll}p_{1} & p_{2} & \cdots & p_{N}\end{array}\right]\left(P\left(q[0]=q_{i} \mid q_{0}\right)=p_{i}\right)$.

The system transition matrix in (1.1) is defined as

$$
\Phi(k ; m)= \begin{cases}\prod_{l=m}^{k-1} A[q[l]], & \text { if } m<k \\ I_{n}, & \text { if } m \geq k\end{cases}
$$

where $I_{n}$ is an identity matrix with the order of $n$. With this matrix, the system's state at time instant $k$ can be expressed as

$$
x[k]=\Phi(k ; 0) x_{0} .
$$

A conditional expectation is defined as

$$
\Phi_{i}[k]=P\left(q[k]=q_{i} \mid q_{0}\right) \mathbf{E}\left[(\Phi(k ; 0))^{[2]} \mid q[k]=q_{i}, q_{0}\right]
$$

where $i=1,2, \ldots, N$. Specially $\Phi_{i}[0]=p_{i} I_{n^{2}}(i=1, \ldots, N)$. Based on the definition of $\Phi_{i}[k]$, we obtain

$$
\mathbf{E}\left[(\Phi(k ; 0))^{[2]} \mid q_{0}\right]=\sum_{i=1}^{N} \Phi_{i}[k] .
$$

By combining all $\Phi_{i}[k](i=1,2, \ldots, N)$ into a bigger matrix, we define

$$
V_{\Phi}[k]=\left[\begin{array}{llll}
\Phi_{1}^{T}[k] & \Phi_{2}^{T}[k] & \cdots & \Phi_{N}^{T}[k]
\end{array}\right]^{T}
$$

Thus, $V_{\Phi}[0]=p^{T} \otimes I_{n^{2}}$.

The necessity proof of Theorem 2.1 needs the following three preliminary Lemmas.

Lemma 2.2. If the jump linear system in (1.1) is mean square stable, then

$$
\lim _{k \rightarrow \infty} \mathrm{E}\left[(\Phi[k ; 0])^{[2]} \mid q_{0}\right]=0, \quad \forall q_{0}
$$


Proof of Lemma 2.2. Because the system is mean square stable, we get

$$
\lim _{k \rightarrow \infty} \mathbf{E}\left[x^{[2]}[k] \mid x_{0}, q_{0}\right]=0, \quad \forall x_{0}, q_{0} .
$$

The expression of $x[k]=\Phi(k ; 0) x_{0}$ yields

$$
\lim _{k \rightarrow \infty} \mathbf{E}\left[\left(\Phi(k ; 0) x_{0}\right)^{[2]} \mid x_{0}, q_{0}\right]=0 .
$$

$\Phi(k ; 0)$ is an $n \times n$ matrix. So we can denote it as $\Phi(k ; 0)=\left[a_{1}(k), a_{2}(k), \ldots, a_{n}(k)\right]$, where $a_{i}(k)$ is a column vector. By choosing $x_{0}=e_{i}\left(e_{i}\right.$ is an $R^{n \times 1}$ vector with the $i$ th element as 1 and the others as 0$),(2.13)$ yields

$$
\lim _{k \rightarrow \infty} \mathbf{E}\left[a_{i}^{[2]}[k] \mid q_{0}\right]=0, \quad i=1,2, \ldots, n .
$$

By the definition of the Kronecker product, we know

$$
(\Phi(k ; 0))^{[2]}=\left[a_{1}[k] \otimes a_{1}[k], \ldots, a_{1}[k] \otimes a_{n}[k], \ldots, a_{n}[k] \otimes a_{1}[k], \ldots, a_{n}[k] \otimes a_{n}[k]\right] .
$$

So (2.14) yields

$$
\lim _{k \rightarrow \infty} \mathbf{E}\left[(\Phi[k ; 0])^{[2]} \mid q_{0}\right]=0, \quad \forall q_{0}
$$

Lemma 2.3. If the jump linear system in (1.1) is mean square stable, then

$$
\lim _{k \rightarrow \infty} \Phi_{i}[k]=0, \quad i=1, \ldots, N, \forall q_{0}
$$

Proof of Lemma 2.3. Choose any $z_{0}, w_{0} \in R^{n}$. Lemma 2.2 guarantees

$$
\lim _{k \rightarrow \infty} \mathbf{E}\left[\left(z_{0}^{[2]}\right)^{T}(\Phi(k ; 0))^{[2]} w_{0}^{[2]} \mid q_{0}\right]=0 .
$$

By the definition of the Kronecker product, we know

$$
\mathbf{E}\left[\left(z_{0}^{[2]}\right)^{T}(\Phi(k ; 0))^{[2]} w_{0}^{[2]} \mid q_{0}\right]=\mathbf{E}\left[\left(z_{0}^{T} \Phi(k ; 0) w_{0}\right)^{2} \mid q_{0}\right] .
$$

By (2.8), (2.9), and (2.19), we get

$$
\mathbf{E}\left[\left(z_{0}^{T} \Phi[k ; 0] w_{0}\right)^{2} \mid q_{0}\right]=\sum_{i=1}^{N} P\left(q[k]=q_{i} \mid q_{0}\right) \mathbf{E}\left[\left(z_{0}^{T} \Phi[k, 0] w_{0}\right)^{2} \mid q[k]=q_{i}, q_{0}\right] .
$$


Because $P\left(q[k]=q_{i} \mid q_{0}\right) \geq 0$ and $\mathbf{E}\left[\left(z_{0}^{T} \Phi[k ; 0] w_{0}\right)^{2} \mid q[k]=q_{i}, q_{0}\right] \geq 0$, the combination of (2.18) and (2.20) yields

$$
\lim _{k \rightarrow \infty} P\left(q[k]=q_{i} \mid q_{0}\right) \mathrm{E}\left[\left(z_{0}^{T} \Phi(k ; 0) w_{0}\right)^{2} \mid q[k]=q_{i}, q_{0}\right]=0
$$

$\Phi(k ; 0)$ is an $n \times n$ matrix. So it can be denoted as $\Phi(k ; 0)=\left(a_{m j}(k)\right)_{m=1, \ldots, n ; j=1, \ldots, n}$. In $(2.21)$, we choose $z_{0}=e_{m}$ and $w_{0}=e_{j}$ and get

$$
\lim _{k \rightarrow \infty} P\left(q[k]=q_{i} \mid q_{0}\right) \mathbf{E}\left[\left(a_{m j}(n)\right)^{2} \mid q[k]=q_{i}, q_{0}\right]=0,
$$

where $i=1,2, \ldots, N, m=1, \ldots, n$ and $j=1, \ldots, n$. By the definition of $\Phi_{i}[k]$, we know the elements of $\Phi_{i}[k]$ take the form of

$$
P\left(q[k]=q_{i} \mid q_{0}\right) \mathbf{E}\left[a_{m_{1} j_{1}}(k) a_{m_{2} j_{2}}(k) \mid q[k]=q_{i}, q_{0}\right],
$$

where $m_{1}, m_{2}, j_{1}, j_{2}=1, \ldots, n$. So $(2.22)$ guarantees

$$
\lim _{k \rightarrow \infty} \Phi_{i}[k]=0, \quad \forall q_{0}
$$

Lemma 2.4. $V_{\Phi}[k]$ is governed by the following dynamic equation

$$
V_{\Phi}[k]=A_{[2]} \quad V_{\Phi}[k-1]
$$

with $V_{\Phi}[0]=p^{T} \otimes I_{n^{2}}$.

Proof of Lemma 2.4. By the definition in (2.8), we can recursively compute $\Phi_{i}[k]$ as follows:

$$
\begin{aligned}
\Phi_{i}[k]= & P\left(q[k]=q_{i} \mid q_{0}\right) \mathbf{E}\left[(A[q[k-1]] \Phi(k-1 ; 0))^{[2]} \mid q[k]=q_{i}, q_{0}\right] \\
= & P\left(q[k]=q_{i} \mid q_{0}\right) \mathbf{E}\left[(A[q[k-1]])^{[2]}(\Phi(k-1 ; 0))^{[2]} \mid q[k]=q_{i}, q_{0}\right] \\
= & P\left(q[k]=q_{i} \mid q_{0}\right) \sum_{j=1}^{N} P\left(q[k-1]=q_{j} \mid q[k]=q_{i}, q_{0}\right) \\
& \times \mathbf{E}\left[(A[q[k-1]])^{[2]}(\Phi(k-1 ; 0))^{[2]} \mid q[k]=q_{i}, q[k-1]=q_{j}, q_{0}\right] \\
= & \sum_{j=1}^{N} A_{j}^{[2]} P\left(q[k]=q_{i} \mid q_{0}\right) P\left(q[k-1]=q_{j} \mid q[k]=q_{i}, q_{0}\right) \\
& \quad \times \mathbf{E}\left[(\Phi(k-1 ; 0))^{[2]} \mid q[k]=q_{i}, q[k-1]=q_{j}, q_{0}\right] .
\end{aligned}
$$


Because $\Phi(k-1 ; 0)$ depends on only $\{q[k-2], q[k-3], \ldots, q[0]\}$ and the jump sequence $\{q[k]\}$ is Markovian, we know

$$
\mathbf{E}\left[(\Phi(k-1 ; 0))^{[2]} \mid q[k]=q_{i}, q[k-1]=q_{j}, q_{0}\right]=\mathbf{E}\left[(\Phi(k-1 ; 0))^{[2]} \mid q[k-1]=q_{j}, q_{0}\right] .
$$

$P\left(q[k]=q_{i} \mid q_{0}\right) P\left(q[k-1]=q_{j} \mid q[k]=q_{i}, q_{0}\right)$ can be computed as

$$
\begin{aligned}
P & \left(q[k]=q_{i} \mid q_{0}\right) P\left(q[k-1]=q_{j} \mid q[k]=q_{i}, q_{0}\right) \\
& =P\left(q[k-1]=q_{j}, q[k]=q_{i} \mid q_{0}\right) \\
& =P\left(q[k]=q_{i} \mid q[k-1]=q_{j}, q_{0}\right) P\left(q[k-1]=q_{j} \mid q_{0}\right) \\
& =P\left(q[k]=q_{i} \mid q[k-1]=q_{j}\right) P\left(q[k-1]=q_{j} \mid q_{0}\right) \\
& =q_{j i} P\left(q[k-1]=q_{j} \mid q_{0}\right) .
\end{aligned}
$$

Substituting (2.27) and (2.28) into the expression of $\Phi_{i}[k]$, we get

$$
\Phi_{i}[k]=\sum_{j=1}^{N} q_{j i} A_{j}^{[2]} \Phi_{j}[k-1] .
$$

After combining $\Phi_{i}[k](i=1,2, \ldots, N)$ into $V_{\Phi}[k]$ as $(2.10)$, we get

$$
V_{\Phi}[k]=A_{[2]} V_{\Phi}[k-1] .
$$

We can trivially get $V_{\Phi}[0]$ from $\Phi_{i}[0]$ by $(2.10)$.

Proof of Necessity of Theorem 2.1. By Lemma 2.3, we get

$$
\lim _{k \rightarrow \infty} V_{\Phi}[k]=0 .
$$

By Lemma 2.4, we get $V_{\Phi}[k]=A_{[2]}^{k} V_{\Phi}[0]$ and $V_{\Phi}[0]=p^{T} \otimes I_{n^{2}}$. Therefore, (2.31) yields

$$
\lim _{k \rightarrow \infty} A_{[2]}^{k}\left(p^{T} \otimes I_{n^{2}}\right)=0,
$$

for any $p$ (the initial distribution of $q_{0}$ ).

$A_{[2]}^{k}$ is an $N n^{2} \times N n^{2}$ matrix. We can write $A_{[2]}^{k}$ as $A_{[2]}^{k}=\left[A^{1}(k), A^{2}(k), \ldots, A^{N}(k)\right]$ where $A^{i}(n)(i=1, \ldots, N)$ is an $N n^{2} \times n^{2}$ matrix. By taking $p_{i}=1$ and $p_{j}=0(j=1, \ldots, i-$ $1, i+1, \ldots, N),(2.32)$ yields

$$
\lim _{k \rightarrow \infty} A^{i}(k)=0
$$

Thus we can get

$$
\lim _{k \rightarrow \infty} A_{[2]}^{k}=0 .
$$

So $A_{[2]}$ is Schur stable. The proof is completed. 


\section{Conclusion}

This paper presents a necessary and sufficient condition for the second moment stability of a discrete-time Markovian jump linear system. Specifically this paper provides proof for the necessity part. Different from the previous necessity proof, this paper confines the state domain to the real space. It investigates the structures of relevant matrices and make a good use of the essential properties of Markov jump linear systems, which may guide the future research on such systems.

\section{Acknowledgment}

This work was supported in part by the National Natural Science Foundation of China (60904012), the Program for New Century Excellent Talents in University (NCET-10-0917) and the Doctoral Fund of Ministry of Education of China (20093402120017).

\section{References}

[1] R. Bellman, Introduction to Matrix Analysis, McGraw-Hill, New York, NY, USA, 1960.

[2] Y. Fang and K. A. Loparo, "Stochastic stability of jump linear systems," IEEE Transactions on Automatic Control, vol. 47, no. 7, pp. 1204-1208, 2002.

[3] O. L. V. Costa and M. D. Fragoso, "Stability results for discrete-time linear systems with Markovian jumping parameters," Journal of Mathematical Analysis and Applications, vol. 179, no. 1, pp. 154-178, 1993.

[4] M. Mariton, Jump Linear Systems in Automatic Control, Marcel Dekker, 1990.

[5] X. Feng, K. A. Loparo, Y. Ji, and H. J. Chizeck, "Stochastic stability properties of jump linear systems," IEEE Transactions on Automatic Control, vol. 37, no. 1, pp. 38-53, 1992.

[6] Y. Ji, H. J. Chizeck, X. Feng, and K. A. Loparo, "Stability and control of discrete-time jump linear systems," Control Theory and Advanced Technology, vol. 7, no. 2, pp. 247-270, 1991.

[7] O. Beldiman, G. C. Walsh, and L. Bushnell, "Predictors for networked control systems," in American Control Conference, pp. 2347-2351, June 2000.

[8] J. Nilsson, Real-time control systems with delays, Ph.D. dissertation, Lund Institute of Technology, 1998.

[9] W. Zhang, M. S. Branicky, and S. M. Phillips, "Stability of networked control systems," IEEE Control Systems Magazine, vol. 21, no. 1, pp. 84-97, 2001.

[10] P. Seiler and R. Sengupta, "Analysis of communication losses in vehicle control problems," in American Control Conference, pp. 1491-1496, June 2001.

[11] B. Lincoln and A. Cervin, "Jitterbug: a tool for analysis of real-time control performance," in IEEE Conference on Decision and Control, pp. 1319-1324, December 2002.

[12] B. Bhaurucha, On the stability of randomly varying systems, Ph.D. dissertation, University of California, Berkeley, Calif, USA, 1961.

[13] Y. Fang, Stability analysis of linear control systems with uncertain parameters, Ph.D. dissertation, Case Western Reserve University, 1994.

[14] W. S. Gray, O. R. Gonzàlez, and S. Patilkulkarni, "Stability of digital control systems subject to jump linear random perturbations," in IEEE Confernce on Decision and Control, pp. 1154-1159, December 2000.

[15] X. Dong, "Stochastic stability of discrete-time jump linear systems," in Chinese Control Conference, 2003.

[16] O. L. V. Costa and M. D. Fragoso, "Comments on: 'Stochastic stability of jump linear systems'," IEEE Transactions on Automatic Control, vol. 49, no. 8, pp. 1414-1416, 2004. 


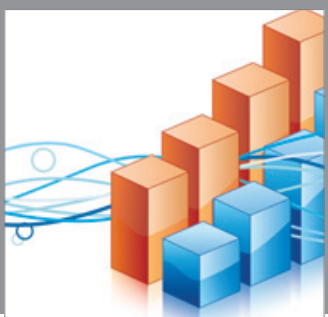

Advances in

Operations Research

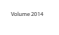

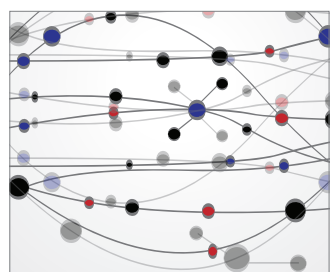

\section{The Scientific} World Journal
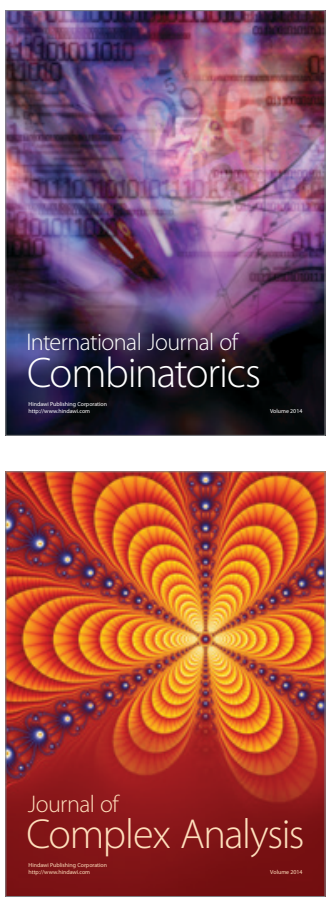

International Journal of

Mathematics and

Mathematical

Sciences
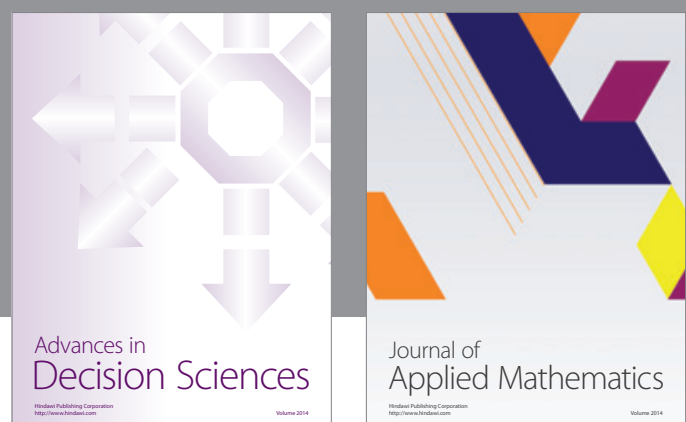

Journal of

Applied Mathematics
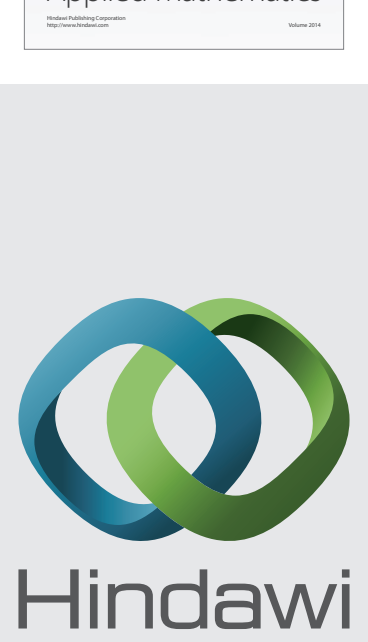

Submit your manuscripts at http://www.hindawi.com
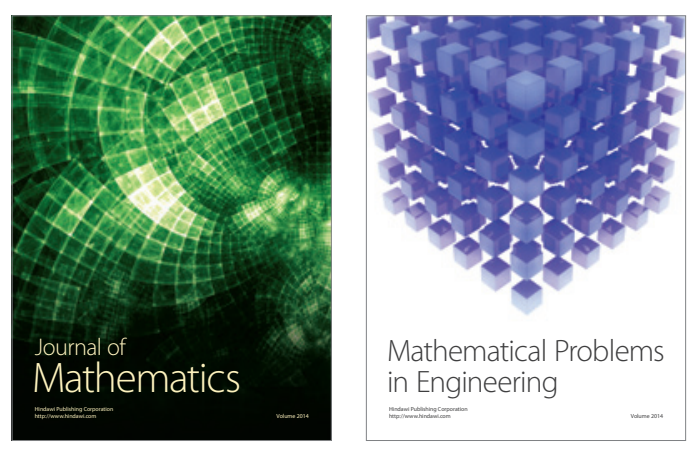

Mathematical Problems in Engineering
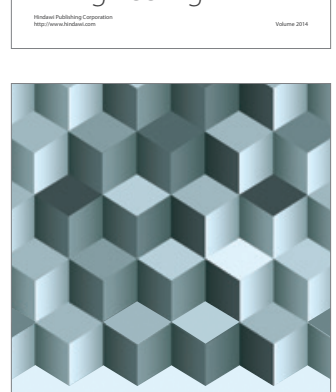

Journal of

Function Spaces
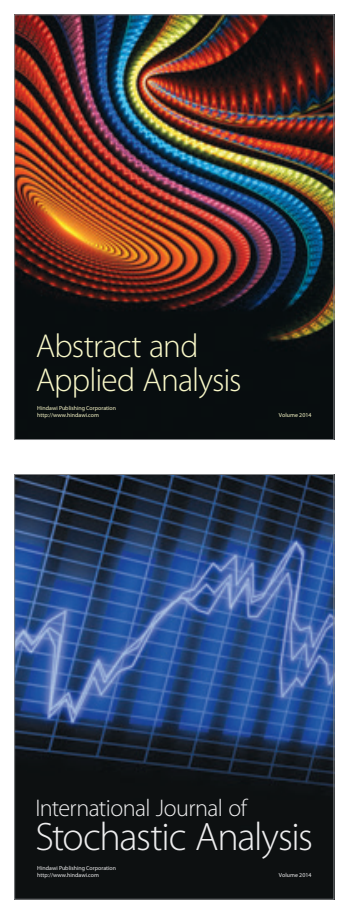

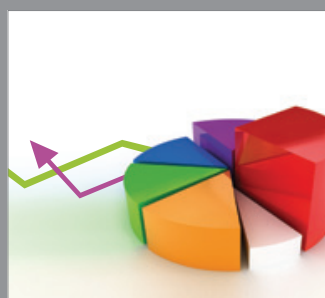

ournal of

Probability and Statistics

Promensencen
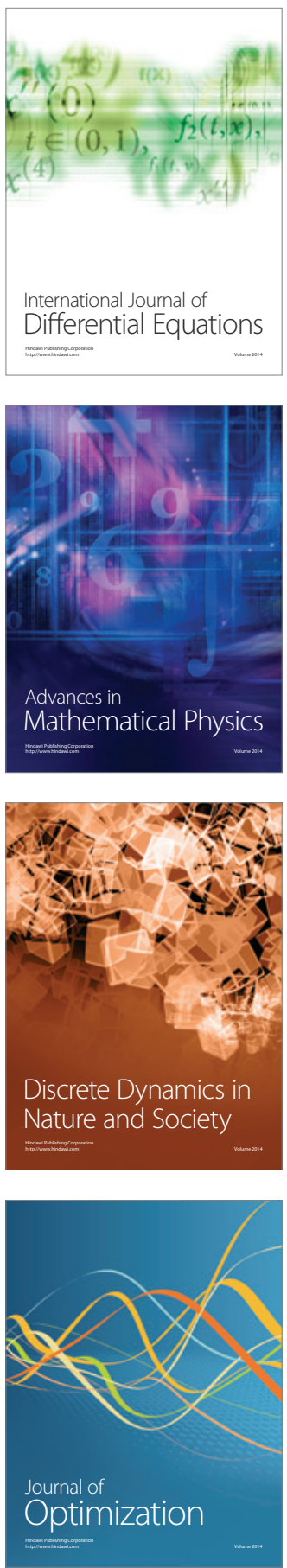